PROCEEDINGS OF THE

AMERICAN MATHEMATICAL SOCIETY

Volume 133, Number 10, Pages 3071-3078

S 0002-9939(05)08063-9

Article electronically published on April 25, 2005

\title{
FINITE SPEED OF PROPAGATION AND LOCAL BOUNDARY CONDITIONS FOR WAVE EQUATIONS WITH POINT INTERACTIONS
}

\author{
PAVEL KURASOV AND ANDREA POSILICANO \\ (Communicated by David S. Tartakoff)
}

\begin{abstract}
We show that the boundary conditions entering in the definition of the self-adjoint operator $\Delta^{A, B}$ describing the Laplacian plus a finite number of point interactions are local if and only if the corresponding wave equation $\ddot{\phi}=\Delta^{A, B} \phi$ has finite speed of propagation.
\end{abstract}

\section{INTRODUCTION AND FRAMEWORK}

The Laplace operator with point interactions in $L^{2}\left(\mathbb{R}^{3}\right)$ can be defined in the following way (see [1, 2] and references therein for more details). Consider any finite set $Y=\left\{y_{j}\right\}_{j=1}^{n}$ of points from $\mathbb{R}^{3}$. Then the restriction $-\Delta_{0}$ of the Laplace operator $-\Delta$ to the set of functions from the Sobolev space $H^{2,2}\left(\mathbb{R}^{3}\right)$ vanishing at all points $y_{j} \in Y$ is a symmetric operator with deficiency indices $(n, n)$. The domain of the adjoint operator $-\Delta_{0}^{*}$ coincides with $H^{2,2}\left(\mathbb{R}^{3} \backslash Y\right)$. Every function $\phi$ from $H^{2,2}\left(\mathbb{R}^{3} \backslash Y\right)$ possesses the following asymptotic representation in a neighborhood of each point $y_{j} \in Y$ :

$$
\phi(x)=\frac{1}{4 \pi\left|x-y_{j}\right|} \phi_{j}^{s}+\phi_{j}^{r}+O\left(\left|x-y_{j}\right|\right), x \rightarrow y_{j},
$$

where the coefficients $\phi_{j}^{r}$ and $\phi_{j}^{s}$ can be considered as certain generalized boundary values of the function $\phi$. These boundary values can be used to describe all selfadjoint extensions of $-\Delta_{0}$ as restrictions of the adjoint operator $-\Delta_{0}^{*}$ to the set of functions satisfying the generalized boundary conditions

$$
A \vec{\phi}^{r}=B \vec{\phi}^{s} .
$$

The $n$-dimensional complex vectors $\vec{\phi}^{s}$ and $\vec{\phi}^{r}$ have coordinates $\phi_{j}^{s}$ and $\phi_{j}^{r}$, respectively, and the $n \times n$ matrices $A, B$ satisfy

$$
A B^{*}=B A^{*}
$$

and

$$
\operatorname{rank}(A, B)=n,
$$

Received by the editors June 4, 2004.

2000 Mathematics Subject Classification. Primary 47B25, 81Q10; Secondary 47A55, 47N50, $81 \mathrm{Q} 15$.

Key words and phrases. Point interactions, singular perturbations, locality, wave equation. 
where $(A, B)$ is the $n \times 2 n$ matrix obtained from $A$ and $B$. The boundary form for the operator $-\Delta_{0}^{*}$

$$
\left\langle\left(-\Delta_{0}^{*}\right) \phi, \psi\right\rangle_{L^{2}}-\left\langle\phi,\left(-\Delta_{0}^{*}\right) \psi\right\rangle_{L^{2}}
$$

determines the following symplectic form in the space $\mathbb{C}^{2 n} \ni \vec{\phi} \equiv\left(\vec{\phi}^{s}, \vec{\phi}^{r}\right)$ of boundary values:

$$
[\vec{\phi}, \vec{\psi}]_{n}:=\left\langle\left(\begin{array}{c}
\vec{\phi}^{r} \\
\vec{\phi}^{s}
\end{array}\right),\left(\begin{array}{cc}
0 & I_{n} \\
-I_{n} & 0
\end{array}\right)\left(\begin{array}{c}
\vec{\psi}^{r} \\
\vec{\psi}^{s}
\end{array}\right)\right\rangle_{\mathbb{C}^{2 n}} .
$$

Then all self-adjoint extensions of $-\Delta_{0}$ can be described by Lagrangian planes associated with this symplectic form. Every such plane $\mathcal{L}$ is described by (2) provided that the matrices $A, B$ satisfy conditions (3) and (44). The first condition guarantees that the symplectic form vanishes for all $\vec{\phi}, \vec{\psi} \in \mathcal{L}$, i.e. that the corresponding extension of $-\Delta_{0}$ is symmetric. The second condition guarantees that the plane $\mathcal{L}$ has the maximal dimension $n$, i.e. that the operator extension is not only symmetric but self-adjoint as well.

Definition 1. The operator $-\Delta^{A, B}$ is the restriction of the adjoint operator $-\Delta_{0}^{*}$ to the domain

$$
D\left(-\Delta^{A, B}\right)=\left\{\phi \in H^{2,2}\left(\mathbb{R}^{3} \backslash Y\right): A \vec{\phi}^{r}=B \vec{\phi}^{s}\right\} .
$$

It is clear that different pairs of matrices $(A, B)$ can determine the same selfadjoint extension of $-\Delta_{0}$. If the matrix $A$ is invertible, then the boundary conditions (2) can be written as

$$
\vec{\phi}^{r}=H \vec{\phi}^{s},
$$

with a Hermitian matrix $H=A^{-1} B$. The operator $-\Delta^{H}$ will be identified with the operator $-\Delta^{I_{n}, H}$.

The action of the operator $-\Delta^{A, B}$ coincides with the action of the (differential) Laplace operator, and therefore this operator is always local, i.e. supp $-\Delta^{A, B} \phi \subseteq$ $\operatorname{supp} \phi$ for any $\phi \in D\left(-\Delta^{A, B}\right)$ and for any admissible pair $A, B$. However the boundary conditions (2) entering in Definition 11 are local, i.e. do not connect the "boundary values" $\phi_{j}^{s}, \phi_{j}^{r}$ at different points $y_{j}$ if and only if the matrices $A$ and $B$ can be chosen diagonal. In this case to check whether the function $\phi$ satisfies the boundary conditions (17) or not, one needs to check the behavior of the function $\phi$ in a certain small neighborhood of each point $y_{j}$ separately. In this case the $n$ dimensional Lagrangian plane $\mathcal{L}$ corresponding to (2) can be presented as a tensor product of $n$ 1-dimensional Lagrangian planes for the symplectic form in $\mathbb{C}^{2}$

$$
\left[\left(\begin{array}{c}
\phi_{j}^{s} \\
\phi_{j}^{r}
\end{array}\right),\left(\begin{array}{c}
\psi_{j}^{s} \\
\psi_{j}^{r}
\end{array}\right)\right]_{1}:=\left\langle\left(\begin{array}{c}
\phi_{j}^{r} \\
\phi_{j}^{s}
\end{array}\right),\left(\begin{array}{cc}
0 & 1 \\
-1 & 0
\end{array}\right)\left(\begin{array}{c}
\psi_{j}^{r} \\
\psi_{j}^{s}
\end{array}\right)\right\rangle_{\mathbb{C}^{2}}
$$

defined for the boundary values $\left(\phi_{j}^{r}, \phi_{j}^{s}\right)$ associated with the point $y_{j}$. Such boundary conditions and corresponding self-adjoint extensions of $-\Delta_{0}$ will be called local.

In fact all interesting local extensions correspond to boundary conditions that can be written in the form (7) with a diagonal matrix $H$. The boundary conditions are local if the matrices $A, B$ can be chosen diagonal. These conditions can be written as (17) if the matrix $A$ is invertible which can be assumed without loss of generality. Indeed if it is not the case, then one can remove a few points from the set $Y$ to get boundary conditions equivalent to (2) with invertible matrix $A$ (maybe having a certain smaller dimension). 
The purpose of this note is to show (see Theorem 4 below) that the boundary conditions (2) are local if and only if the wave equation

$$
\ddot{\phi}-\Delta^{A, B} \phi=0
$$

has finite speed of propagation. We recall that in general an abstract wave equation

$$
\ddot{\phi}+\mathcal{A} \phi=0
$$

is said to have finite speed of propagation if for any solution $\phi$ and for any real $t$,

$$
\operatorname{diam}(\operatorname{supp} \phi(t)) \leq 2 v|t|+\operatorname{diam}(\operatorname{supp} \phi(0) \cup \operatorname{supp} \dot{\phi}(0))
$$

holds with a certain $v \in(0, \infty)$. While it is obvious what "local boundary conditions" means in the case of $-\Delta^{A, B}$, the situation is not so clear for self-adjoint operators arising from extensions of symmetric operators obtained by restricting the Laplacian to the set of smooth functions with compact support on $\mathbb{R}^{3} \backslash M$, where $M$ is not discrete, for example a subset with Hausdorff dimension $0<d<2$ or a low-dimensional manifold [2, 8]. Since the wave equation (10) is always well defined (in the sense that it generates a strongly continuous group of evolution) for any bounded from below self-adjoint operator $\mathcal{A}$ (see e.g. 3], chapter 2, section 7), our result could be used to shed light on the problem of the locality of boundary conditions in more complicated situations.

We conclude by pointing out that locality of boundary conditions, hence finite speed of propagation, is equivalent to locality in the sense of forms. According to [9], section 1.2, the self-adjoint operator $-\Delta^{A, B}$ is said to be form-local if the sesquilinear form of the operator $-\Delta^{A, B}$ vanishes on any two functions from the domain $D\left(F^{A, B}\right)$ of the quadratic form having disjoint supports. Theorem 2 from 7] implies that the operator $-\Delta^{A, B}$ is form local if and only if the boundary conditions (2) can be written in the form (7) with local (i.e. diagonal) operator $H$. Hence the boundary conditions (77) with diagonal matrices $H$ describe all form-local operators.

\section{Proofs}

The following representation for functions from the domain of the operator $-\Delta^{A, B}$ will be used in our proofs.

We denote by $\bar{H}^{1,2}\left(\mathbb{R}^{3}\right)$ the homogeneous Sobolev space of tempered distributions $\phi$ such that $i \nabla \phi$ is square integrable. Then $\bar{H}^{2,2}\left(\mathbb{R}^{3}\right)$ denotes the space of $\phi \in \bar{H}^{1,2}\left(\mathbb{R}^{3}\right)$ such that $-\Delta \phi \in L^{2}\left(\mathbb{R}^{3}\right)$. In general neither $\phi \in \bar{H}^{1,2}\left(\mathbb{R}^{3}\right)$ nor $\phi \in \bar{H}^{2,2}\left(\mathbb{R}^{3}\right)$ imply $\phi \in L^{2}\left(\mathbb{R}^{3}\right)$; by Sobolev embedding theorems one has $\bar{H}^{1,2}\left(\mathbb{R}^{3}\right) \subset L^{6}\left(\mathbb{R}^{3}\right)$ and $\bar{H}^{2,2}\left(\mathbb{R}^{3}\right) \subset C_{b}\left(\mathbb{R}^{3}\right)$.

We use the symbol $\mathcal{G}$ to denote the Green function of $-\Delta$, i.e.

$$
\mathcal{G}(x):=\frac{1}{4 \pi} \frac{1}{|x|} .
$$

For any $y_{j} \in Y$ we define the functions

$$
\mathcal{G}_{j}(x):=\mathcal{G}\left(x-y_{j}\right), \quad d_{j}(x):=\left|x-y_{j}\right|,
$$

and the symmetric matrices $G=\left(G_{i j}\right)$ and $D=\left(d_{i j}\right), 1 \leq i, j \leq n$, by

$$
G_{i j}:=\mathcal{G}\left(y_{i}-y_{j}\right), i \neq j, G_{j j}:=0, \quad d_{i j}:=\left|y_{i}-y_{j}\right| .
$$


Lemma 2. The self-adjoint operator $-\Delta^{A, B}$ given in Definition 1 can be re-written in the following way:

$$
\begin{aligned}
& D\left(-\Delta^{A, B}\right):=\left\{\phi \in L^{2}\left(\mathbb{R}^{3}\right): \phi=\phi_{0}+\sum_{1 \leq j \leq n} \zeta_{\phi}^{j} \mathcal{G}_{j}, \phi_{0} \in \bar{H}^{2,2}\left(\mathbb{R}^{3}\right),\right. \\
& \left.\vec{\zeta}_{\phi} \in \mathbb{C}^{n}, \sum_{1 \leq j \leq n} A_{i j} \phi_{0}\left(y_{j}\right)=\sum_{1 \leq j \leq n}(B-A G)_{i j} \zeta_{\phi}^{j}\right\}, \\
& -\Delta^{A, B} \phi:=-\Delta \phi_{0} .
\end{aligned}
$$

Proof. It is sufficient to note that for any $\phi$ from the operator domain defined above one has $\phi \in H^{2,2}\left(\mathbb{R}^{3} \backslash Y\right)$ and

$$
\lim _{x \rightarrow y_{j}}\left(\phi-\zeta_{\phi}^{j} \mathcal{G}_{j}\right)(x)=\phi_{0}\left(y_{j}\right)+\sum_{1 \leq k \leq n} G_{j k} \zeta_{\phi}^{k} .
$$

Thus

$$
\phi_{j}^{s}=\zeta_{\phi}^{j}, \quad \phi_{j}^{r}=\phi_{0}\left(y_{j}\right)+\sum_{1 \leq k \leq n} G_{j k} \zeta_{\phi}^{k}
$$

The proof of our main result relies on the following representation of the solutions of the Cauchy problem given by the wave equation (2). This result, in the simpler case of a single point interaction, was already obtained, by a different, less explicit proof, in [5] (also see [6]).

Theorem 3. The Cauchy problem

$$
\begin{gathered}
\ddot{\phi}=\Delta^{A, B} \phi, \\
\phi(0) \in D\left(\Delta^{A, B}\right), \\
\dot{\phi}(0) \in D\left(F^{A, B}\right)
\end{gathered}
$$

has a unique strong solution

$$
\phi \in C\left(\mathbb{R} ; D\left(\Delta^{A, B}\right) \cap C^{1}\left(\mathbb{R} ; D\left(F^{A, B}\right)\right) \cap C^{2}\left(\mathbb{R} ; L^{2}\left(\mathbb{R}^{3}\right)\right)\right.
$$

explicitly given, when $t \geq 0$, by

$$
\phi(t)=\phi_{f}(t)+\sum_{1 \leq j \leq n} \theta\left(t-d_{j}\right) \zeta_{\phi}^{j}\left(t-d_{j}\right) \mathcal{G}_{j},
$$

where $\theta$ denotes the Heaviside function, $\phi_{f}$ is the unique solution of the Cauchy problem for the free wave equation

$$
\begin{aligned}
& \ddot{\phi}_{f}=\Delta \phi_{f}, \\
& \phi_{f}(0)=\phi(0), \\
& \dot{\phi}_{f}(0)=\dot{\phi}(0),
\end{aligned}
$$

and $\vec{\zeta}_{\phi}(t), t \geq 0$, is the unique solution of the Cauchy problem for the system of inhomogeneous retarded first-order differential equations

$$
\begin{aligned}
& \sum_{\substack{1 \leq j \leq n \\
\zeta_{\phi}(0)=\zeta_{\phi(0)}}} A_{i j}\left(\frac{\dot{\zeta}_{\phi}^{j}}{4 \pi}-\phi_{f}\left(y_{j}\right)\right)+B_{i j} \zeta_{\phi}^{j}=\sum_{1 \leq j, k \leq n} A_{i j} G_{j k} \theta\left(\cdot-d_{j k}\right) \zeta_{\phi}^{k}\left(\cdot-d_{j k}\right) \\
&
\end{aligned}
$$


Proof. Since $-\Delta^{A, B}$ is a bounded from below self-adjoint operator, it is well known from the theory of abstract wave equations in Hilbert spaces (see e.g. [3], chapter 2, section 7) that the corresponding Cauchy problem has a unique strong solution in $C\left(\mathbb{R} ; D\left(\Delta^{A, B}\right) \cap C^{1}\left(\mathbb{R} ; D\left(F^{A, B}\right)\right) \cap C^{2}\left(\mathbb{R} ; L^{2}\left(\mathbb{R}^{3}\right)\right)\right.$. Let us denote by $\phi(t)$ such a solution. By the structure of $D\left(-\Delta^{A, B}\right)$ given in Lemma 2 we know that

$$
\phi(t)=\phi_{0}(t)+\sum_{1 \leq j \leq n} \zeta_{\phi}^{j}(t) \mathcal{G}_{j}
$$

with $\phi_{0}(t) \in \bar{H}^{2,2}\left(\mathbb{R}^{3}\right)$ and

$$
\sum_{1 \leq j \leq n} A_{i j} \phi_{0}\left(t, y_{j}\right)=\sum_{1 \leq j \leq n}(B-A G)_{i j} \zeta_{\phi}^{j}(t)
$$

for all $t \in \mathbb{R}$. Let us now define, for any $t \geq 0$,

$$
\phi_{f}(t):=\phi(t)-\sum_{1 \leq j \leq n} \phi_{j}(t)
$$

where $\phi_{j}(t)$ denotes the spherical wave

$$
\phi_{j}(t):=\theta\left(t-d_{j}\right) \zeta_{\phi}^{i}\left(t-d_{j}\right) \mathcal{G}_{j}
$$

Since

$$
\ddot{\phi}_{j}=\Delta \phi_{j}+\zeta_{\phi}^{j} \delta_{y_{j}}
$$

one has

$$
\begin{aligned}
\ddot{\phi}_{f} & =\Delta\left(\phi_{0}-\sum_{1 \leq j \leq n} \phi_{j}\right)-\sum_{1 \leq j \leq n} \zeta_{\phi}^{j} \delta_{y_{j}} \\
& =\Delta\left(\phi_{0}-\sum_{1 \leq j \leq n}\left(\phi_{j}-\zeta_{\phi}^{j} \mathcal{G}_{j}\right)\right) \\
& =\Delta\left(\phi-\sum_{1 \leq j \leq n} \phi_{j}\right) \\
& =\Delta \phi_{f}
\end{aligned}
$$

and thus $\phi_{f}$ is the unique solution of the Cauchy problem (13), (14), (15). Writing the boundary conditions (18) by using the decompositions of $\phi$ given by the relation 
(19), one obtains

$$
\begin{aligned}
& \sum_{1 \leq j, k \leq n}\left(B_{i j}-A_{i k} G_{k j}\right) \zeta_{\phi}^{j}(t) \\
= & \sum_{1 \leq j \leq n} A_{i j}\left(\lim _{x \rightarrow y_{j}}\left(\phi(t)-\sum_{1 \leq k \leq n} \zeta_{\phi}^{k}(t) \mathcal{G}_{k}\right)(x)\right) \\
= & \sum_{1 \leq j \leq n} A_{i j}\left(\phi_{f}\left(t, y_{j}\right)+\sum_{1 \leq k \leq n} \theta\left(t-d_{j k}\right) G_{j k} \zeta_{\phi}^{k}\left(t-d_{j k}\right)\right. \\
& \left.-\sum_{1 \leq k \leq n} G_{j k} \zeta_{\phi}^{k}(t)+\lim _{x \rightarrow y_{j}} \frac{\zeta_{\phi}^{j}\left(t-\left|y_{j}-x\right|\right)-\zeta_{\phi}^{j}(t)}{4 \pi\left|y_{j}-x\right|}\right) \\
= & \sum_{1 \leq j \leq n} A_{i j}\left(\phi_{f}\left(t, y_{j}\right)+\sum_{1 \leq k \leq n} \theta\left(t-d_{j k}\right) G_{j k} \zeta_{\phi}^{k}\left(t-d_{j k}\right)\right. \\
& \left.-\sum_{1 \leq k \leq n} \zeta_{\phi}^{j} G_{j k}-\frac{1}{4 \pi} \dot{\zeta}_{\phi}^{j}(t)\right) .
\end{aligned}
$$

This shows that $\vec{\zeta}_{\phi}$ is a solution of (16), (17). Such a Cauchy problem has a unique solution. Indeed let $\vec{\zeta}(t)$, with $\vec{\zeta}(0)=\overrightarrow{0}$, solve the system

$$
\frac{1}{4 \pi} A \dot{\vec{\zeta}}+B \vec{\zeta}=0 \text {. }
$$

By (3) and $\operatorname{Ker}(A) \cap \operatorname{Ker}(B)=\{\overrightarrow{0}\}$, which is a consequence of (4), one has

$$
|\operatorname{det}(i A+B)|^{2}=\operatorname{det}\left((i A+B)\left(-i A^{*}+B^{*}\right)\right)=\operatorname{det}\left(A A^{*}+B B^{*}\right) \neq 0,
$$

so that $z A+B, z \in \mathbb{C}$, is a regular pencil of matrices, i.e. $p(z):=\operatorname{det}(z A+B)$ is not the zero polynomial. By Theorem 3.2 of $[4$ this implies $\vec{\zeta}(t)=0$.

The above theorem has an analogous version for negative times. In this case one obtains a representation involving the advanced waves $\theta\left(-t-d_{j}\right) \zeta_{\phi}^{i}\left(t+d_{j}\right) \mathcal{G}_{j}$, instead of the retarded waves $\theta\left(t-d_{j}\right) \zeta_{\phi}^{i}\left(t-d_{j}\right) \mathcal{G}_{j}$. Since there is no substantial difference between these two situations, in the proof of the following theorem we will consider only the positive time case, the proof in the negative time case being essentially the same.

The previous theorem shows that compactly supported Cauchy data always gives rise to the compactly supported solution. However this does not necessarily imply a finite speed of propagation. Indeed we have the following

Theorem 4. Let $-\Delta^{A, B}$ be a point perturbation of the Laplace operator described by Definition 11 with the matrices $A, B$ satisfying (3) and (4). Then the wave equation

$$
\ddot{\phi}-\Delta^{A, B} \phi=0
$$

has finite velocity of propagation if and only if $-\Delta^{A, B}$ is defined by local boundary conditions, i.e. if and only if both matrices $A$ and $B$ can be chosen diagonal simultaneously. 
Proof. Let $\phi(t)$ be a solution of (12) with $\operatorname{diam}\left(S_{0}\right)<+\infty$, where

$$
S_{0}:=\operatorname{supp} \phi(0) \cup \operatorname{supp} \dot{\phi}(0) \text {. }
$$

By Theorem [3,

$$
\phi(t)=\phi_{f}(t)+\sum_{1 \leq i \leq n} \phi_{i}(t),
$$

where $\phi_{i}(t)$ denotes the spherical wave

$$
\phi_{i}(t):=\theta\left(t-d_{i}\right) \zeta_{\phi}^{i}\left(t-d_{i}\right) G_{i} .
$$

Since the free wave equation has velocity of propagation equal to one, we have (here and below $t \geq 0$ )

and

$$
\operatorname{diam}\left(\operatorname{supp} \phi_{f}(t)\right) \leq 2 t+\operatorname{diam}\left(S_{0}\right)
$$

$$
\operatorname{diam}\left(\operatorname{supp} \phi_{i}(t)\right)=\operatorname{diam}\left(\left\{d_{i}<t\right\}\right)=2 t .
$$

Thus, if $Y \subseteq S_{0}$,

$$
\operatorname{diam}(\operatorname{supp} \phi(t)) \leq 2 t+\operatorname{diam}\left(S_{0}\right) .
$$

Let us now consider the case $Y_{0}:=Y \backslash\left(S_{0} \cap Y\right) \neq \emptyset$.

Suppose that $A$ and $B$ can be chosen diagonal. Let $y_{i} \in Y_{0}$. By (16), in order for the wave $\phi_{i}(t)$ to be present, the point $y_{i}$ must have been reached at some earlier stage either by the free wave $\phi_{f}$ or by a spherical wave $\phi_{j}$ originated from a different point $y_{j}$. Since all these waves travel with the speed $v=1$, in conclusion one has that

$$
\operatorname{diam}(\operatorname{supp} \phi(t)) \leq 2 t+\operatorname{diam}\left(S_{0}\right) .
$$

We have proven sufficiency.

To prove the necessity consider the operator $-\Delta^{A, B}$ described by arbitrary matrices $A, B$ satisfying (3) and (4). Take an arbitrary point $y_{k} \in Y$ and initial data satisfying the following conditions:

$$
\begin{array}{r}
\vec{\zeta}_{\phi_{0}} \equiv \vec{\zeta}_{\phi}(0)=0, \\
\operatorname{dist}\left(y_{k}, S_{0}\right)<\operatorname{dist}\left(Y \backslash\left\{y_{k}\right\}, S_{0}\right) .
\end{array}
$$

Then the solution $\phi_{f}$ of (13), (14), (15) reaches the point $y_{k}$ before it reaches any other point from $Y$. Therefore, for some sufficiently small $\epsilon 0$ and for any $t$ such that $\operatorname{dist}\left(y_{k}, S_{0}\right)<t<\operatorname{dist}\left(y_{k}, S_{0}\right)+\epsilon$, the system of equations (16) takes the form

$$
\sum_{1 \leq j \leq n} \frac{1}{4 \pi} A_{i j} \dot{\zeta}_{\phi}^{j}+B_{i j} \zeta_{\phi}^{j}=A_{i k} \phi_{f}\left(y_{k}\right), \quad i=1, \ldots, n .
$$

The evolution has finite speed of propagation only if all $\zeta_{\phi}^{i}, i \neq k$ are zero, otherwise

$$
\operatorname{diam}(\operatorname{supp} \phi(t))\left|y_{i}-y_{k}\right|, \quad \operatorname{dist}\left(y_{k}, S_{0}\right)<t<\operatorname{dist}\left(y_{k}, S_{0}\right)+\epsilon,
$$

which violates (11) since both $\operatorname{diam}\left(S_{0}\right)$ and $\operatorname{dist}\left(y_{k}, S_{0}\right)$ can be made arbitrarily.

If all $\zeta_{\phi}^{i}$ are zero for $i \neq k$, then (23) becomes

$$
\frac{1}{4 \pi} A_{i k} \dot{\zeta}_{\phi}^{k}+B_{i k} \zeta_{\phi}^{k}=A_{i k} \phi_{f}\left(y_{k}\right), \quad i=1, \ldots, n,
$$

so that the $k$-th columns of $A$ and $B$ are linearly dependent. Since the point $y_{k}$ is chosen arbitrarily from $Y$, finite speed of propagation implies that the linear spaces $V_{k}$ spanned by the $k$-th columns of $A$ and $B$ are one dimensional for all $k$. Then condition (44) implies that any vector $v=\sum_{k=1}^{n} v_{k}, v_{k} \in V_{k}$, is equal to zero only if all $v_{k}=0$. Therefore the corresponding Lagrangian plane is a tensor product of 
Lagrangian planes for boundary values associated with each singular point $y_{k}$, i.e. that the boundary conditions are local.

\section{REFERENCES}

[1] S. Albeverio, F. Gesztesy, R. Høegh-Krohn, and H. Holden, Solvable Models in Quantum Mechanics, Berlin, Heidelberg, New York, Springer-Verlag, 1988. MR 0926273 (90a:81021)

[2] S. Albeverio and P.Kurasov, Singular Perturbations of Differential Operators, Cambridge Univ. Press, 2000. MR1752110 (2001g:47084)

[3] J.A. Goldstein, Semigroups of Linear Operators and Applications, Oxford Univ. Press, 1985. MR0790497 (87c:47056)

[4] J.W. Hooker and C.E. Langenhop, On Regular Systems of Linear Differential Equations with Constant Coefficients, Rocky Mountain J. Math., 12 (1982), 591-614. MF 0683854(84d:34008)

[5] D. Noja and A.Posilicano, The Wave Equation with One Point Interaction and the (Linearized) Classical Electrodynamics of a Point Particle, Ann. Inst. H. Poincaré Phys. Theor., 68 (1998), 351-377. MR 1622543 (99b:78006)

[6] D. Noja and A. Posilicano, Delta Interactions and Electrodynamics of Point Particles, CMS Conf. Proc., 29 (2000), 505-516. MR1803443 (2001j:78011)

[7] K.V.Pankrashkin, Locality of Quadratic Forms for Point Perturbations of Schrödinger Operators, Math. Notes, 70 (2001), 384-391. MF 1882252 (2002k:47033)

[8] B.S. Pavlov, Boundary conditions on thin manifolds and the semiboundedness of the threebody Schrödinger operator with point potential, Math. Sb. (N.S.), 136(178) (1988), 163-177. MR0954922 (90g:35120)

[9] Yu.G. Shondin, Semibounded Local Hamiltonians in $\mathbb{R}^{4}$ for a Laplacian Perturbed on Curves with Corner Points, Theoret. and Math. Phys., 106 (1996), 151-166. MF1402004(97g:47044)

Department of Mathematics, Lund Institute of Technology, P.O. Box 118, 22100 LUND, SWEDEN

E-mail address: kurasov@maths.lth.se

Dipartimento di Scienze, Università dell'Insubria, I-22100 Como, Italy

E-mail address: posilicano@uninsubria.it 